\title{
Multidrug Therapy and Evolution of Antibiotic Resistance: When Order Matters
}

\author{
Gabriel G. Perron, ${ }^{a, b}$ Sergey Kryazhimskiy, ${ }^{a}$ Daniel P. Rice, ${ }^{a}$ and Angus Buckling ${ }^{b, c}$ \\ FAS Center for Systems Biology and Department of Organismic and Evolutionary Biology, Harvard University, Cambridge, Massachusetts, USA ; Department of Zoology, \\ University of Oxford, Oxford, United Kingdom ${ }^{\text {; }}$; and Biosciences, University of Exeter, Tremough, Cornwall, United Kingdom ${ }^{c}$
}

\begin{abstract}
The evolution of drug resistance among pathogenic bacteria has led public health workers to rely increasingly on multidrug therapy to treat infections. Here, we compare the efficacy of combination therapy (i.e., using two antibiotics simultaneously) and sequential therapy (i.e., switching two antibiotics) in minimizing the evolution of multidrug resistance. Using in vitro experiments, we show that the sequential use of two antibiotics against Pseudomonas aeruginosa can slow down the evolution of multiple-drug resistance when the two antibiotics are used in a specific order. A simple population dynamics model reveals that using an antibiotic associated with high costs of resistance first minimizes the chance of multidrug resistance evolution during sequential therapy under limited mutation supply rate. As well as presenting a novel approach to multidrug therapy, this work shows that costs of resistance not only influences the persistence of antibiotic-resistant bacteria but also plays an important role in the emergence of resistance.
\end{abstract}

W ith the rapid emergence of microorganisms resistant to one or many antibiotics (24), clinicians worldwide have increasingly relied on multidrug therapy to fight bacterial infections (21, 22). Although multidrug treatments proved successful in reducing the prevalence of severe infections, such as Mycobacterium tuberculosis $(10,14)$, the pervasive use of antibiotics has resulted in the evolution of multidrug resistance (MDR) in many species of bacteria $(1,26)$. MDR is now frequent in many health care-associated bacterial infections, such as Clostridium difficile, Staphylococcus aureus, and Pseudomonas aeruginosa (19, 21, 25), raising serious challenges regarding the optimal use of multidrug therapy (15).

Two key objectives of multidrug therapy are to minimize the rate of evolution of multidrug-resistant bacteria and to limit the total amount of antibiotic used in hospitals (17). These problems can be approached at two levels: the hospital-wide level and the single-patient level. At the hospital-wide level, two therapeutic approaches are typically employed: mixing therapy (i.e., two or more drugs are used simultaneously in the hospital, where each patient receives a single drug) and periodic hospital-wide rotation of antibiotics (i.e., two or more drugs can be alternated periodically within a hospital). While clinical trials for different bacterial infections produced mixed results $(4,8,33,34,40)$, theoretical results typically suggest that a mixing strategy minimizes the evolution of MDR compared to that of hospital-wide rotation $(9,11$, 42). The mixing strategy is believed to increase the rate at which bacteria are exposed to different antibiotics relative to rotation, therefore minimizing the opportunity for resistance evolution. Although recent theoretical results suggest that it should be possible to find an optimal rotation strategy that minimizes resistance evolution (6), the range of optimal parameters is a matter of debate $(5,11,20)$.

Despite the use of hospital-wide strategies, pathogenic bacteria, such as $P$. aeruginosa and $M$. tuberculosis, often evolve resistance in the course of a single-host treatment $(16,33)$. Therefore, clinicians also face decisions on how to best administer one or many antibiotics to a single patient (18). For example, antibiotics can be used simultaneously (i.e., combination therapy) or sequen- tially (i.e., sequential therapy), where two or more different antibiotics are used one after the other. While combination therapy has been used successfully, for example, to treat Helicobacter pylori, the etiological agent of peptic ulcers, combination therapy can be associated with uncomfortable side effects (13), or its effectiveness may be limited as a result of poorly studied drug interactions (41).

Unlike hospital-wide cycling, sequential therapy within a single host exposes bacterial infections to a rapid change in antibiotics. While the cycling of antibiotics within a hospital system can take months to years to implement, it is possible to switch antibiotics within a single host over a matter of days. Provided that the antibiotics chosen for sequential therapy elicit no cross-resistance, mutants resistant against one antibiotic are unlikely to reach high frequencies within the host before a second antibiotic is applied. Furthermore, pleiotropic fitness costs associated with resistance mutations are believed to affect the persistence of antibiotic-resistant bacteria and to slow the spread of resistance (2). Therefore, a rapid switch in antibiotic use has the potential to minimize multidrug resistance while mitigating negative clinical consequences of combination therapy.

Given that the rate at which resistance mutations are generated varies among antibiotics (22) and that fitness costs associated with resistance mutations vary both within and among individual antibiotics $(2,28)$, the success of sequential therapy is likely to depend on both the antimicrobial activity of the antibiotics and the order in which they are used. Building from the work presented in Perron et al. (28), we investigate the importance of antibiotic treatment order in determining the efficacy of multidrug therapy

Received 2 April 2012 Accepted 18 June 2012

Published ahead of print 22 June 2012

Address correspondence to Gabriel G. Perron, gperron@fas.harvard.edu.

G.G.P. and S.K. contributed equally to this work.

Copyright @ 2012, American Society for Microbiology. All Rights Reserved.

doi:10.1128/AEM.01078-12 
using experimental and theoretical results. First, we compared the effect of combination therapy and sequential therapy on MDR evolution in experimental populations of $P$. aeruginosa maintained under different mutation supply rate regimes. We show that using two antibiotics one after the other early in the treatment can give results similar to those obtained by using the two drugs simultaneously; but the effect depends on which antibiotic is used first and on the mutation supply rate of resistant mutations in the population. Second, we introduce a simple population dynamics model identifying pleiotropic fitness costs associated with resistance mutations as a plausible mechanism responsible for the order effect observed in our experiment.

\section{MATERIALS AND METHODS}

Study organism and growth conditions. In this experiment, we cultured a total of 72 experimental populations of Pseudomonas aeruginosa strain PAO1 (37). P. aeruginosa is a nonrecombinogenic Gram-negative bacterium that is an important opportunistic pathogen of human. Each population was initially inoculated with approximately $10^{4}$ cells and was incubated at $37^{\circ} \mathrm{C}$ in $150 \mu \mathrm{l}$ of $\mathrm{M} 9 \mathrm{~KB}$ medium (per liter: $20 \mathrm{~g}$ proteose peptone no. 3, $12.8 \mathrm{~g} \mathrm{Na}_{2} \mathrm{HPO}_{4} \cdot 7 \mathrm{H}_{2} \mathrm{O}, 10 \mathrm{~g}$ glycerol, $3 \mathrm{~g} \mathrm{KH}_{2} \mathrm{PO}_{4}, 0.5 \mathrm{~g} \mathrm{NaCl}$, and $1 \mathrm{~g} \mathrm{NH}_{4} \mathrm{Cl}$ ). Every $24 \mathrm{~h}$, each culture was mixed thoroughly by pipetting 50 $\mu \mathrm{l}$ in and out repeatedly before $1 \%$ of the overnight culture (approximately $10^{6}$ bacterial cells) was transferred to a fresh microcosm. Growth was monitored daily for 10 days and was measured as an optical density at $600 \mathrm{~nm}\left(\mathrm{OD}_{600}\right)$ using a universal microplate reader (BioTek, Winooski, VT). Three antibiotic treatments were implemented as described below.

Mutation supply rate. We looked at the effect of increasing the mutation supply rate by manipulating the immigration rate of susceptible bacteria sampled from an isogenic population of $P$. aeruginosa grown overnight. For each antibiotic treatment, we grew 12 replicate populations with the following mutation supply rates: no immigration and $0.1 \%$ (approximately 1,000 cells), $1.0 \%$ (approximately 10,000 cells), and 10\% (approximately 100,000 cells) immigration.

Antibiotic treatments. The antibiotics rifampin and streptomycin were used in this experiment. Rifampin acts by lodging itself into the DNA/RNA tunnel of the polymerase and sterically blocking elongation of nascent mRNA molecules (12). Streptomycin targets the S16 rRNA protein of the $30 \mathrm{~S}$ ribosomal subunit, interfering with the binding of tRNA and therefore initiation of protein synthesis (36). Previous studies of rifampin- and streptomycin-resistant mutants of $P$. aeruginosa showed that cross-resistance between the two drugs is unlikely in similar experimental conditions $(28,39)$.

The medium was supplemented with the antibiotics at the following concentrations: rifampin, $62.5 \mu \mathrm{g} \cdot \mathrm{ml}^{-1}$; streptomycin, $16 \mu \mathrm{g} \cdot \mathrm{ml}^{-1}$. These concentrations of antibiotics were shown to completely inhibit the growth of $P$. aeruginosa in the absence of immigration (28). Also, we established that rifampin was more effective than streptomycin at inhibiting the growth of $P$. aeruginosa and that resistance to rifampin incurred a higher fitness cost (28). For each mutation supply rate treatment, three different combinations of the antibiotics were used to treat populations of P. aeruginosa: (i) the combination of streptomycin and rifampin, (ii) sequential therapy with rifampin used first, and (iii) sequential therapy with streptomycin used first. During sequential therapy, antibiotics were switched at every transfer.

Pleiotropic fitness costs of resistance. At the end of the experiment, the populations were grown for three transfers in unsupplemented $\mathrm{KB}$ to test the heritability of resistance and to estimate the pleiotropic cost of resistance of each population. The cost of resistance was measured as the optical density $\left(\mathrm{OD}_{600}\right)$ in unsupplemented $\mathrm{KB}$ and was compared to that of PAO1 strains cultivated in parallel for the length of the experiment to control for adaptation to normal laboratory conditions.

Statistical analyses. To analyze the evolution of antibiotic resistance during the course of the experiment, we modeled the temporal dynamics of bacterial growth using a hierarchical linear mixed model (the lme function of the nlme package of the R 2.11.1 software), sometimes referred to as repeated-measure analysis of variance (ANOVA). We used the optical density data $\left(\mathrm{OD}_{600}\right)$ as the response. Time (number of transfers following the beginning of the experiment) was considered a random variable, while antibiotic treatment ( 3 levels) and the rate of immigration (4 levels) were considered fixed effects. We also accounted for the nonlinear growth dynamics over the bacteria populations by computing the quadratic term for time. Because all replicates were started under similar conditions, we constrained the model to a unique intercept. Replicates were taken to be random effects and were nested within treatments. We began by fitting the full model that included all fixed effects and their interactions and then simplified it by sequential backward selection. We used an F-test to compare the fit of different models $\left[F_{(i, j)}\right.$ denotes the $F$ statistic with $i$ and $j$ degrees of freedom]. A variance function (varIdent of the nlme library) that permits different variances for each level of a stratification variable (here, treatment) was used to model heteroscedasticity when necessary. We also used the corAR1 function to model the autocorrelation structure in the time series. The significance of fixed effects was tested with F-tests. Differences between treatments were tested with pairwise comparisons using log likelihood ratio tests. Model parameters and confidence intervals were estimated with restricted maximum likelihood methods (31).

We also modeled the effect of antibiotic treatments and mutation supply rate on the average growth $\left(\mathrm{OD}_{600}\right)$ of all selection lines over the length of the experiment. The model was fit in a linear general model using antibiotic treatments (3 levels) as a fixed factor and mutation supply rate as a continuous factor (this is to control for the nonnormality of the data). Finally, we looked at the effect of antibiotic treatment (3 levels) and mutation supply rate (4 levels) on pleiotropic fitness costs of resistance, fitting the carrying capacity of each population in the absence of antibiotic as a dependent variable. All analyses and model assumptions were performed and verified using R 2.10.1 software (http://www.r-project.org).

\section{RESULTS}

Experimental evolution of multidrug resistance under multidrug therapy. We investigated the efficacy of multidrug therapy and sequential therapy by treating experimental populations of the bacterium $P$. aeruginosa with one of following three treatments: (i) the combination of streptomycin and rifampin, (ii) sequential therapy of rifampin and streptomycin with rifampin used as the first antibiotic, and (iii) sequential therapy of rifampin and streptomycin with streptomycin used as the first antibiotic. We observed that the three treatments have significantly different effects on MDR evolution and that these effects depend on the mutation supply rate of the treated population (treatment $\times$ immigration $X$ time $\times$ time: $F_{(6,624)}=5.266 ; P<0.0001$; Fig. 1$)$. Under normal growth conditions, we found that MDR evolved only in populations first exposed to streptomycin during sequential therapy; all populations treated with both antibiotics went extinct after $24 \mathrm{~h}$, while populations treated with rifampin first went extinct after being subsequently exposed to streptomycin (Fig. 1A). Given that $P$. aeruginosa can evolve resistance to both rifampin and streptomycin when used individually (28), our results confirm that, given a certain order, sequential therapy can significantly decrease resistance evolution.

Bacterial populations rarely evolve in isolation (33). In previous studies, we showed that immigration of susceptible bacterial cells from a larger source population could favor adaptation to antibiotic environments by increasing the supply of resistance mutations in local populations of bacteria (27-29). For this reason, we simulated the effect of immigration by supplementing each experimental population treated with antibiotics with susceptible bacterial cells at each transfer. As predicted, immigration 

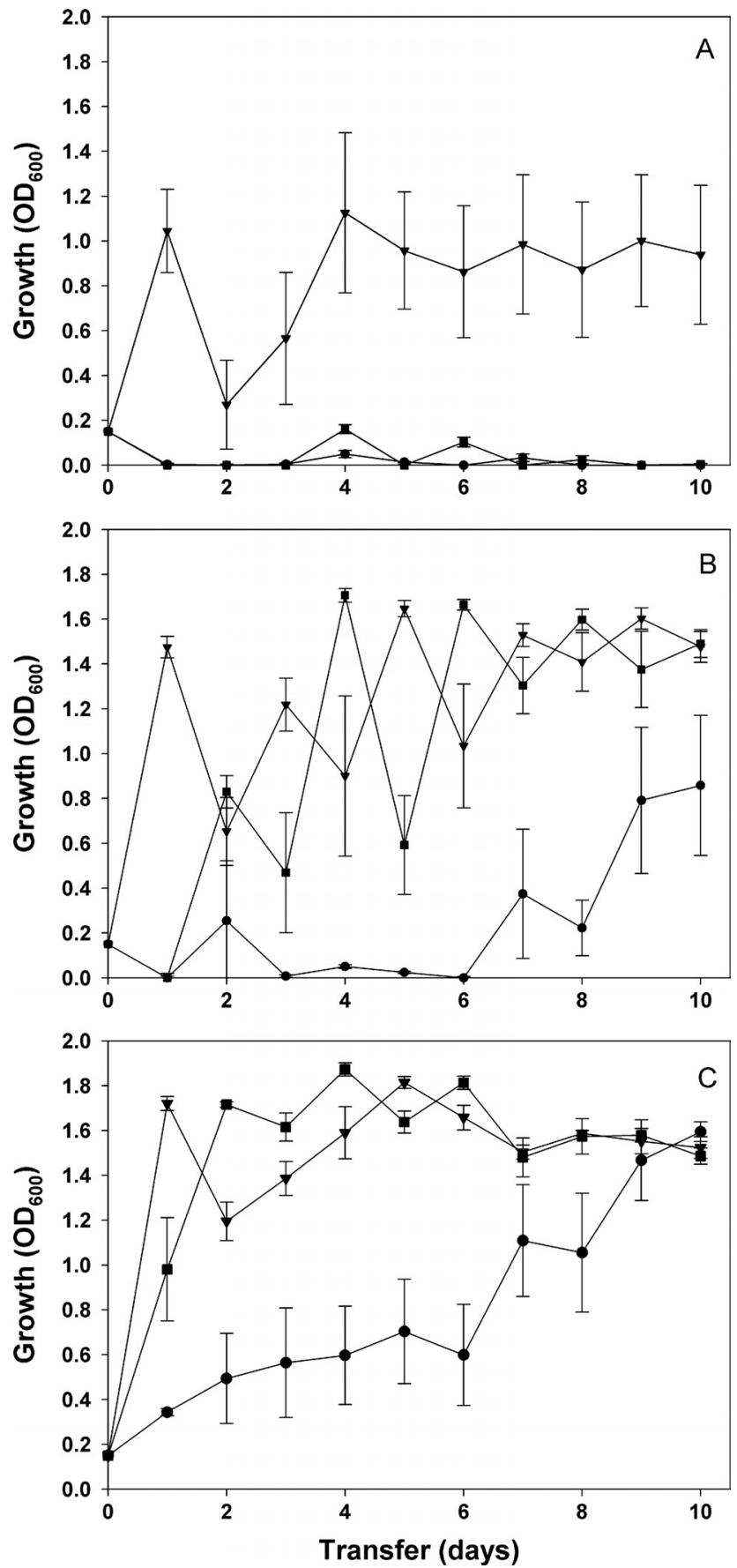

FIG 1 Evolution of multidrug resistance in experimental populations of Pseudomonas aeruginosa treated with multidrug therapy. Multidrug treatments were as follows: rifampin and streptomycin simultaneously (filled circles), sequential therapy with exposure to streptomycin first (filled triangles), and sequential therapy with exposure to rifampin first (filled squares). For sequential therapy, the antibiotics were switched on a daily basis. Populations were grown in the absence of immigration (A), or with $1 \%$ (B) and 10\% (C) immigration. Resistance was measured as average density $\left(\mathrm{OD}_{600}\right)$ of the selection lines at the end of each transfer. Error bars represent standard errors of the means.

had a positive effect on MDR evolution (immigration, $F_{(3,60)}=$ 44.306; $P<0.0001$ ) while small amounts of immigration (i.e., 1,000 susceptible cells per transfer) favored MDR evolution under sequential therapy (Fig. 1B) and a higher level of immigration (i.e.,

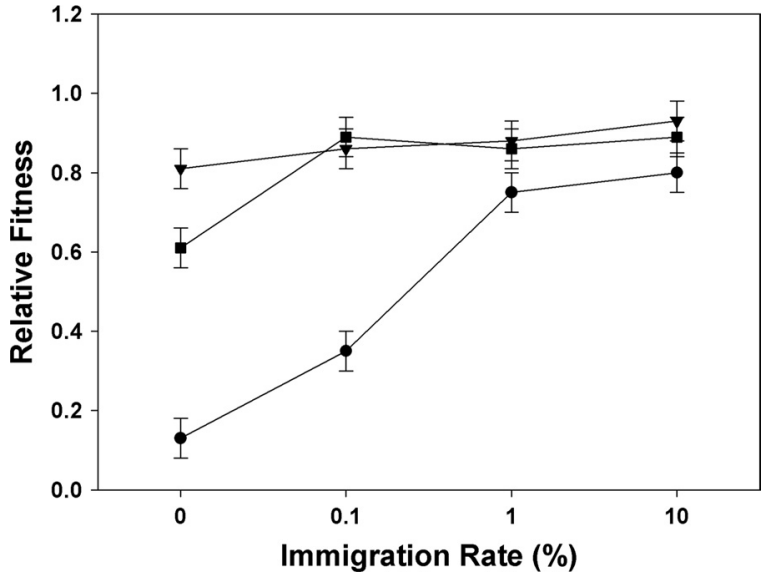

FIG 2 Pleiotropic fitness costs of experimental populations of Pseudomonas aeruginosa that have evolved resistance under three multidrug treatments and four immigration rates. Drug treatments are as follow: rifampin and streptomycin simultaneously (filled circle), sequential therapy with exposure to streptomycin first (filled triangles), and sequential therapy with exposure to rifampin first (filled squares). Relative fitness is measured as the growth $\left(\mathrm{OD}_{600}\right)$ of the resistant populations in the absence of drug compared to that of control populations grown in the absence of drug for the duration of the experiment.

100,000 cells per transfer) and allowed MDR evolution in all treatments (Fig. 1C).

Pleiotropic fitness costs of resistance. To assess whether the type of multidrug therapy used impacted the pleiotropic fitness costs associated with MDR, we measured the growth of each MDR population in the absence of antibiotics. We found that the choice of therapy and the mutation supply rate regimen significantly affected the evolution of costs of resistance (treatment $\times$ immigration: $F_{(1,66)}=24.578 ; P<0.001$; Fig. 2). While fitness cost of resistance decreased as immigration rate increased, fitness costs were generally higher under combination therapy than under both sequential treatments. At lower immigration rates, costs of resistance were generally lower in sequential therapy initiated with streptomycin than in sequential therapy initiated with rifampin. As immigration increased, both sequential treatments generated little or no cost of resistance. These results indicate that the combination and the order in which two antibiotics are used over the course of a treatment plays an important role in the evolution of resistance costs in addition to resistance evolution.

Modeling the antibiotic order effect in sequential therapy. To understand the effect of antibiotic treatment order on the evolution of resistance under sequential therapy, we considered a simple population dynamics model of the evolution of MDR in a bacterial population that is sequentially exposed to two antibiotics. Because most extinction events in our experiment happened within the first two antibiotic switches, we focused on the population dynamic involved in the first two transfers. In this model, we assume that bacteria acquire resistance mutations against the first and the second antibiotics with rates $\mu_{1}$ and $\mu_{2}$, respectively, and that an antibiotic-resistant allele confers a cost of resistance in the absence of that antibiotic. In particular, mutations that confer resistance against the first antibiotic are deleterious with selection coefficient $s_{1}$ in the absence of this antibiotic, while the fitness cost of mutation conferring resistance to the second antibiotic is $s_{2}$. We also assume that $s_{i} \gg \mu_{j}, i, j=1,2$, which reflects our knowledge of typical mutation rates and costs of resistance (22). 
In the first phase of our experiment, the population grows in the absence of antibiotics and reaches population size $N_{0}$. We assume that the fraction of mutants resistant to the first antibiotic by the end of this phase reaches the mutation-selection balance, $\mu_{1} / s_{1}$, and that there are no MDR mutants. Thus, by the time the first antibiotic treatment is applied, there are on average $N_{0} \mu_{1} / s_{1}$ bacterial cells that harbor resistance to the first antibiotic. Starting with this (relatively small) number of resistant cells, the population then grows for time $t$ in the presence of the first antibiotic, until the second antibiotic is applied and the first antibiotic is withdrawn. We consider that MDR evolves in our experiment if on average there is at least one cell harboring resistance to both antibiotics at the time the second antibiotic is applied. Mathematically, if $N_{12}(t)$ is the expected number of MDR cells at time $t$ during the second growth phase, the equation for MDR evolution is

$$
N_{12}(T) \geq 1
$$

To express equation 1 in terms of evolutionary parameters $\mu_{1}$, $\mu_{2}, s_{1}$, and $s_{2}$, we assume that in the presence of the first antibiotic, the population stays below its carrying capacity and grows exponentially for time $T$. If $N_{1}(t)$ is the expected number of bacteria resistant to the first antibiotic but not to the second antibiotic at time $t$, the average population dynamics is described by differential equations

$$
\begin{gathered}
\dot{N}_{1}=r_{1} N_{1}-\mu_{2} N_{1}, \\
\dot{N}_{12}=\left(r_{1}-s_{2}\right) N_{12}+\mu_{2} N_{1}
\end{gathered}
$$

with the condition at the beginning of the second growth phase of

$$
\begin{gathered}
N_{1}(0)=N_{0} \mu_{1} / s_{1}, \\
N_{12}(0)=0
\end{gathered}
$$

where $r_{1}$ is the intrinsic growth rate of single-drug-resistant bacteria in the presence of the first antibiotic. The solution to equations 2 and 3 is

$$
\begin{gathered}
N_{1}(t)=\left(N_{0} \mu_{1} / s_{1}\right) e^{\left(r_{1}-\mu_{2}\right) t} \\
N_{12}(t)=\frac{\left(N_{0} \mu_{1} / s_{1}\right) \mu_{2}}{\mu_{2}-s_{2}}\left(e^{\left(r_{1}-s_{2}\right) t}-e^{\left(r_{1}-\mu_{2}\right) t}\right)
\end{gathered}
$$

Equation 1 then becomes

$$
\frac{N_{0} \mu_{1} \mu_{2} e^{\left(r_{1}-\mu_{2}\right) t}}{s_{1}} \frac{1-e^{-\left(s_{2}-\mu_{2}\right) t}}{s_{2}-\mu_{2}} \geq 1
$$

which we can rewrite as

$$
s_{1} \leq A f\left(s_{2}\right)
$$

where

$$
A=N_{0} \mu_{1} \mu_{2} e^{\left(r_{1}-\mu_{2}\right) t} t
$$

and

$$
f(s)=\frac{1-e^{-\left(s-\mu_{2}\right) t}}{\left(s-\mu_{2}\right) t}
$$

Notice that function $f(s)$ is positive (because, as mentioned above, in practice $s_{2} \gg \mu_{2}$ ) and is monotonically decreasing.

We now ask which antibiotic should be applied first, given that the costs of resistance to each are different. Consider two antibiotics, $X$ and $Y$, for which resistance incurs the costs $s_{X}$ and $s_{Y}$,

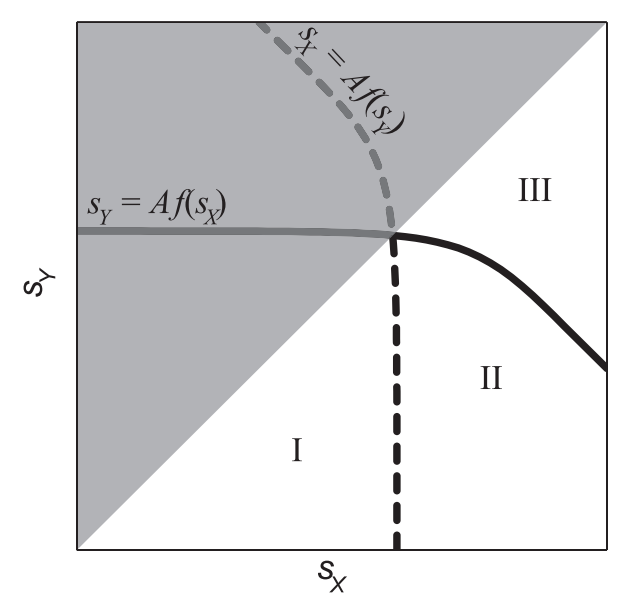

FIG 3 Schematic illustration of regions where MDR is expected to evolve under different treatments. Any pair of antibiotics $X$ and $Y$ with costs of resistance $s_{X}$ and $s_{Y}$ (such that $s_{X}>s_{Y}$ ) represents a point in the lower semiplane of the $s_{X}, s_{Y}$ plane. The region to the left of the dashed line is the region where MDR is expected to evolve under the strong-weak treatment (i.e., when antibiotic $X$ is applied first). The region below the solid line is the region where MDR is expected to evolve under the weak-strong treatment (i.e., when antibiotic $Y$ is applied first). The parameter region where MDR evolves under the strong-weak treatment (region I) is strictly smaller than the region where MDR evolves under weak-strong treatment (regions I and II). Thus, the strong-weak treatment represents a safer bet against MDR evolution under uncertainty.

respectively. Without loss of generality, we can assume that $s_{X}>$ $s_{Y}$. We then say that antibiotic $X$ is in this sense "strong" and antibiotic $Y$ is "weak." From equation 4, the equation of MDR evolution in case $X$ is applied before $Y$ ("strong-weak" treatment) is

$$
s_{X} \leq A f\left(s_{Y}\right)
$$

and, vice versa, in case $Y$ is applied before $X$ ("weak-strong" treatment), MDR evolves if

$$
s_{Y} \leq A f\left(s_{X}\right)
$$

As illustrated in Fig. 3, the inequalities of equations 5 and 6 divide the $\left(s_{X}, s_{Y}\right)$ semiplane into three regions. As we mentioned above, we consider only the area under the diagonal ( where $s_{X}>s_{Y}$ is respected). In region I, both equations 5 and 6 are satisfied, implying that MDR is expected to evolve regardless of the order in which antibiotics are applied. In region II, equation 6 is satisfied but equation 5 is not, which implies that MDR is expected to evolve only under the weak-strong treatment. Finally, in region III, both equations 5 and 6 are violated, and MDR is not expected to evolve under either treatment. In this analysis, the parameter region where MDR evolves under the weak-strong treatment (regions I and II) is strictly larger than the parameter region where MDR evolves under the strong-weak treatment (region I). In other words, our model predicts that MDR is less likely to evolve under sequential therapy when the cost of resistance to the first antibiotic used is higher than that of the second antibiotic used.

Predictions of our simple model are consistent with our experimental observations. As shown previously in Perron et al. (28), resistance mutations against rifampin generally confer a larger cost on fitness than resistance mutations against streptomycin. In the above-described terminology, rifampin is the strong antibiotic and streptomycin is the weak antibiotic. The fact that the MDR evolves under the weak-strong treatment (streptomycin first) but 
not under the strong-weak treatment (rifampin first) suggests that the costs of resistance against these antibiotics fall into region II of the parameter space of our experiment (Fig. 3).

\section{DISCUSSION}

In this study, we show that the order in which two antibiotics are used during sequential therapy of a single bacterial infection has a significant effect on MDR evolution and its fitness costs. When we grew our experimental populations in isolation, without the influx in mutation provided by the immigration of cells from a source population, the use of rifampin before streptomycin precluded the evolution of MDR just as successfully as the combination of the two antibiotics. Using a simple population dynamics model, we demonstrate that the fitness cost associated with mutations conferring resistance to the first antibiotic predominantly determines this order effect. Specifically, the chance of MDR evolution is reduced by first using the antibiotic for which resistant mutation confers the highest fitness cost. Our results demonstrate that costs of resistance play an important role in reducing the frequency of resistant mutants in bacteria populations and therefore minimizing the rate of MDR evolution. Given that our model captures the fundamental population parameters governing resistance evolution, we expect that our results are not limited to the specific antibiotics used but instead may be general to many antibiotic combinations.

Our model provides a simple explanation for why antibiotics' order in sequential treatment can affect the evolution of resistance. Because of severe competition in large bacterial populations within the host prior to the application of any antibiotics, the frequency of resistant mutants is limited by the rate at which they arise and the pleiotropic fitness costs they carry. Resistance mutations that arise at a low probability and resistance mutations that incur a large cost on fitness will be less frequent in a population that is not treated with antibiotics, a phenomenon known as the mutation-selection balance (7). Importantly, at the moment the first antibiotic is applied, (i) the frequency of a resistant mutant is inversely proportional to the cost of resistance that it carries, and (ii) the probability that this mutant spreads in the population after the antibiotic is applied is proportional to its initial frequency. This implies that the cost of resistance against the first antibiotic directly modulates bacterial population density during the antibiotic-free phase and, consequently, the probability of MDR evolution. The application of the first antibiotic dramatically reduces population density and, thus, competition between bacterial cells. With little competition, the rate of resistance evolution against the second antibiotic is limited primarily by the rate at which such resistance mutations arise; the cost of resistance against the second antibiotic plays a relatively small role. Mathematically, this is reflected in that the shape of function $f\left(s_{2}\right)$ in equation 4 depends very weakly on $s_{2}$. Therefore, if resistance mutations against the two antibiotics incur different costs, the order in which the antibiotics are applied critically determines the probability of MDR evolution.

Although mutation supply rate is an important factor in the evolution of MDR, it does not contribute to the effect of antibiotic treatment order in our model. This result means that changes in mutation rates over time within a population, such as hypermutability arising (29) or one of the drugs inducing the bacterium's SOS response (32), will not affect our conclusions. The model provides a striking match with our experimental results: MDR evolves under sequential therapy when streptomycin was first applied but did not when rifampin was the first antibiotic used. Since resistance to rifampin generally confers a larger cost on fitness than resistance to streptomycin (28), the order effect observed in this study is consistent with our theoretical model and is likely to be independent of the target and the function of the antibiotics.

Two important assumptions reflecting the settings of our experimental work are crucial for our theoretical results to hold. First, the time scale between the use of the two antibiotics determines the frequency of resistance mutations found at each phase. While the preantibiotic phase must be long enough for the population to reach the mutation-selection balance with respect for resistance mutations, the population size posttreatment must remain low relative to carrying capacity; otherwise, the resistance cost against the second antibiotic would become important. Although it can be difficult to control the exact concentration of antibiotics that reach the site of infection (3), it is relatively simple to switch between different antibiotics over a short period of time when treating individual patients.

Second, we make the assumption that there is no cross-resistance, no recombination between the resistance mutations, no possibility of compensatory mutations, and no epistasis between resistance mutations. Cross-resistance between the two antibiotics would cancel the order effect since evolution of resistance to one antibiotic would confer resistance to the second antibiotic. With the accumulation of data on resistance and cross-resistance for an increasing number of antibiotics, however, it should be possible for clinicians to avoid the use of two drugs for which cross-resistance is known. Horizontal gene transfer can bring together resistance genes that evolved in different bacterial lineages (23) and can therefore promote MDR evolution (30). The compensation of fitness costs associated with resistance could also contribute to increasing the frequency of antibiotic-resistant mutants in natural populations of bacteria by alleviating the competitive disadvantage of resistant mutants $(29,35)$. Finally, epistatic interactions among different resistance mutations could either promote or inhibit the evolution of MDR (38).

Given the availability of minimal information pertaining to fitness costs associated with resistance to specific antibiotics, our results provide a simple guideline for sequential multidrug-therapy: one ought to use the antibiotic incurring the stronger cost of resistance as the first-line antibiotic in order to minimize the change of MDR evolution. Because of the different pharmacodynamics characteristics associated with different antibiotics and the complex nature of bacterial infections in the human body, it is practically impossible to accurately model the evolution of antibiotic resistance within a human host. Still, our simple model captures the key aspects of such evolution and makes a strong qualitative prediction: MDR is less likely to evolve if the antibiotic that incurs larger resistance costs is applied first. Although costs of resistance to specific antibiotics can change substantially between bacterial species and between different environments $(2,27,28)$, it usually follows predictable distributions readily identified in laboratory experiments (22).

As the use of multidrug therapy is increasingly prevalent in the treatment of health care-associated infections such as $P$. aeruginosa (21), it is crucial to understand the full evolutionary consequences associated with drug deployments. Our results not only demonstrate the influence cost of resistance has on the evolution 
of MDR but suggest a simple approach that could potentially improve multidrug therapy.

\section{ACKNOWLEDGMENTS}

We thank Sam P. Brown, R. Fredrick Inglis, and Luiz-Miguel Chevin for their comments on earlier versions of the manuscript.

This work was funded by the European Research Council (ERC) and the Leverhulme Trust. G.G.P. was funded during this work by the Clarendon Funds of the University of Oxford and the National Science Engineering and Research Council of Canada (NSERC) and is now funded by the Fond Québécois pour la Recherche sur la Nature et les Technologies (FQRNT).

The funders had no role in study design, data collection and analysis, decision to publish, or preparation of the manuscript.

\section{REFERENCES}

1. Albert TJ, et al. 2005. Mutation discovery in bacterial genomes: metronidazole resistance in Helicobacter pylori. Nat. Methods 2:951-953.

2. Andersson DI, Hughes D. 2010. Antibiotic resistance and its cost: is it possible to reverse resistance? Nat. Rev. Microbiol. 8:260-271.

3. Andes D, Anon J, Jacobs MR, Craig WA. 2004. Application of pharmacokinetics and pharmacodynamics to antimicrobial therapy of respiratory tract infections. Clin. Lab. Med. 24:477-502.

4. Bal AM, Kumar A, Gould IM. 2010. Antibiotic heterogeneity: from concept to practice, p 81-91. In Bush K (ed), Antimicrobial therapeutics reviews, vol 1213. Wiley-Blackwell, Malden, MA.

5. Beardmore RE, Peña-Miller R. 2010. Antibiotic cycling versus mixing: the difficulties of using mathematical models to definitively quantify their relative merits. Math. Biosci. Eng. 7:923-933.

6. Beardmore RE, Peña-Miller R. 2010. Rotating antibiotics selects optimally against antibiotic resistance, in theory. Math. Biosci. Eng. 7:26.

7. Bell G. 2008. Selection: the mechanism of evolution. Oxford University Press, Oxford, United Kingdom.

8. Bennett KM, et al. 2007. Implementation of antibiotic rotation protocol improves antibiotic susceptibility profile in a surgical intensive care unit. J. Trauma 63:307-311.

9. Bergstrom CT, Lo M, Lipsitch M. 2004. Ecological theory suggests that antimicrobial cycling will not reduce antimicrobial resistance in hospitals. Proc. Natl. Acad. Sci. U. S. A. 101:13285-13290.

10. Bhusal Y, Shiohira CM, Yamane N. 2005. Determination of in vitro synergy when three antimicrobial agents are combined against Mycobacterium tuberculosis. Int. J. Antimicrob. Agents 26:292-297.

11. Bonhoeffer S, Wiesch PA, Kouyos RD. 2010. Rotating antibiotics does not minimize selection for resistance. Math. Biosci. Eng. 7:919-922.

12. Campbell EA, et al. 2001. Structural mechanism for rifampicin inhibition of bacterial RNA polymerase. Cell 104:901-912.

13. Chiba N, Rao BV, Rademaker JW, Hunt RH. 1992. Metaanalysis of the efficacy of antibiotic-therapy in eradicating Helicobacter-pylori. Am. J. Gastroenterol. 87:1716-1727.

14. Daniels M, Hill AB. 1952. Chemotherapy of pulmonary tuberculosis in young adults; an analysis of the combined results of three Medical Research Council trials. Br. Med. J. 1:1162-1168.

15. Eber MR, Laxminarayan R, Perencevich EN, Malani A. 2010. Clinical and economic outcomes attributable to health care-associated sepsis and pneumonia. Arch. Intern. Med. 170:347-353.

16. Gagneux S, et al. 2006. The competitive cost of antibiotic resistance in Mycobacterium tuberculosis. Science 312:1944-1946.

17. Gould IM, van der Meer JWM (ed). 2007. Antibiotic policies: fighting resistance, 1st ed. Springer Science+Business Media, LLC, New York, NY.

18. Hauser AR. 2007. Antibiotics basics for clinicians: choosing the right antibacterial agent. Lippincott Williams \& Wilkins, Baltimore, MD.
19. Kerr KG, Snelling AM. 2009. Pseudomonas aeruginosa: a formidable and ever-present adversary. J. Hosp. Infect. 73:338-344.

20. Kouyos RD, zur Wiesch PA, Bonhoeffer S. 2011. Informed switching strongly decreases the prevalence of antibiotic resistance in hospital wards. PLoS Comput. Biol. 7:e1001094. doi:10.1371/journal.pcbi.1001094.

21. Levy SB, Marshall B. 2004. Antibacterial resistance worldwide: causes, challenges and responses. Nat. Med. 10(Suppl 12):S122-S129.

22. MacLean RC, Hall AR, Perron GG, Buckling A. 2010. The population genetics of antibiotic resistance: integrating molecular mechanisms and treatment contexts. Nat. Rev. Genet. 11:405-414.

23. Ochman H, Lawrence JG, Grolsman EA. 2000. Lateral gene transfer and the nature of bacterial innovation. Nature 405:299-304.

24. Palumbi SR. 2001. Humans as the world's greatest evolutionary force. Science 293:1786-1790.

25. Pearson A. 2009. Historical and changing epidemiology of healthcareassociated infections. J. Hosp. Infect. 73:296-304.

26. Perron GG, Bell G, Quessy S. 2008. Parallel evolution of multidrugresistance in Salmonella enterica isolated from swine. FEMS Microbiol. Lett. 281:17-22.

27. Perron GG, Gonzalez A, Buckling A. 2008. The rate of environmental change drives adaptation to an antibiotic sink. J. Evol. Biol. 21:1724-1731.

28. Perron GG, Gonzalez A, Buckling A. 2007. Source-sink dynamics shape the evolution of antibiotic resistance and its pleiotropic fitness cost. Proc. Biol. Sci. 274:2351-2356.

29. Perron GG, Hall AR, Buckling A. 2010. Hypermutability and compensatory adaptation in antibiotic-resistant bacteria. Am. Nat. 176:303-3011.

30. Perron GG, Lee AE, Wang Y, Huang WE, Barraclough TG. 2012. Bacterial recombination promotes the evolution of multidrug-resistance in functionally diverse populations. Proc. Biol. Sci. 279:1477-1484.

31. Pinheiro JC, Bates DM. 2000. Mixed-effects models in S and S-PLUS. Springer, New York, NY.

32. Radman M. 1975. SOS repair hypothesis: phenomenology of an inducible DNA repair which is accompanied by mutagenesis. Basic Life Sci. 5A:355367.

33. Reinhardt A, et al. 2007. Development and persistence of antimicrobial resistance in Pseudomonas aeruginosa: a longitudinal observation in mechanically ventilated patients. Antimicrob. Agents Chemother. 51:13411350 .

34. Sandiumenge A, et al. 2006. Impact of diversity of antibiotic use on the development of antimicrobial resistance. J. Antimicrob. Chemother. 57: 1197-1204.

35. Schrag SJ, Perrot V, Levin BR. 1997. Adaptation to the fitness costs of antibiotic resistance in Escherichia coli. Proc. R. Soc. Biol. Sci. B 264:12871291.

36. Sharma D, Cukras AR, Rogers EJ, Southworth DR, Green R. 2007. Mutational analysis of S12 protein and implications for the accuracy of decoding by the ribosome. J. Mol. Biol. 374:1065-1076.

37. Stover CK, et al. 2000. Complete genome sequence of Pseudomonas aeruginosa PAO1, an opportunistic pathogen. Nature 406:959-964.

38. Trindade S, et al. 2009. Positive epistasis drives the acquisition of multidrug resistance. PLoS Genet. 5:e1000578. doi:10.1371/journal.pgen.1000578.

39. Ward H, Perron GG, Maclean RC. 2009. The cost of multiple drug resistance in Pseudomonas aeruginosa. J. Evol. Biol. 22:997-1003.

40. Warren DK, et al. 2004. Cycling empirical antimicrobial agents to prevent emergence of antimicrobial-resistant Gram-negative bacteria among intensive care unit patients. Crit. Care Med. 32:2450-2456.

41. Yeh P, Tschumi AI, Kishony R. 2006. Functional classification of drugs by properties of their pairwise interactions. Nat. Genet. 38:489-494.

42. zur Wiesch PA, Kouyos R, Engelstädter J, Regoes RR, Bonhoeffer S. 2011. Population biological principles of drug-resistance evolution in infectious diseases. Lancet Infect. Dis. 11:236-247. 